Pacific Journal of Mathematics

ZERO DIVISORS AND GROUP YON NEUMANN ALGEBRAS 


\title{
ZERO DIVISORS AND GROUP VON NEUMANN ALGEBRAS
}

\author{
P. A. LINNELL
}

Let $G$ be a discrete group, let $L^{2}(G)$ denote the Hilbert space with Hilbert basis the elements of $G$, and let $W(G)$ denote the group von Neumann algebra of $G$. The class of elementary amenable groups is the smallest class of groups which contains all abelian and all finite groups, is extension closed, and is closed under directed unions. If $G$ is an elementary amenable group whose finite subgroups have bounded order, $\alpha$ is a nonzero divisor in $\mathbb{C} G$, and $\beta$ is a nonzero element of $L^{2}(G)$, we shall prove $\alpha \beta \neq 0$. We shall also consider the quotient rings of $\mathbb{C} G$ and $W(G)$.

1. Introduction. Let $G$ be a group and let $L^{2}(G)$ denote the Hilbert space with Hilbert basis $\{g \mid g \in G\}$. Thus $L^{2}(G)$ consists of all formal sums $\sum_{g \in G} a_{g} g$ where $a_{g} \in \mathbb{C}$ and $\sum_{g \in G}\left|a_{g}\right|^{2}<\infty$. If $\alpha=\sum_{g \in G} a_{g} g \in \mathbb{C} G$ (so $a_{g} \in \mathbb{C}$ and $a_{g}=0$ for all but finitely many $g$ ) and $\beta=\sum_{g \in G} b_{g} g \in L^{2}(G)$, then

$$
\alpha \beta=\sum_{g, h \in G} a_{g} b_{h} g h=\sum_{g \in G}\left(\sum_{h \in G} a_{g h^{-1}} b_{h}\right) g
$$

is a well-defined element of $L^{2}(G)$. In this paper we consider the following conjecture:

Conjecture 1. If $G$ is a torsion free group, $\alpha \in \mathbb{C} G, \beta \in L^{2}(G)$, and $\alpha, \beta \neq 0$, then $\alpha \beta \neq 0$.

Results on this have been obtained in $[6,20,21]$. Recall that the class of elementary amenable groups is the smallest class of groups which contains all finite and abelian groups, is extension closed, and is closed under directed unions (see $[5,13]$ ). It is also closed under subgroups and quotient groups, and contains all solvable by finite groups. On the other hand any group which contains a subgroup isomorphic to a nonabelian free group is not elementary amenable. We shall prove

THEOREM 2. If $G$ is a torsion free elementary amenable group, $\alpha \in \mathbb{C} G, \beta \in L^{2}(G)$, and $\alpha, \beta \neq 0$, then $\alpha \beta \neq 0$. 
In [20] Rosset needed a special case of Theorem 2 to prove a vanishing theorem for Euler characteristics. Recall that the group $G$ is of type $F P$ if the trivial $\mathbb{Z} G$-module $\mathbb{Z}$ has a resolution

$$
0 \rightarrow P_{n} \rightarrow P_{n-1} \rightarrow \cdots \rightarrow P_{1} \rightarrow P_{0} \rightarrow \mathbb{Z} \rightarrow 0
$$

where the $P_{i}$ are finitely generated projective $\mathbb{Z} G$-modules. Then the Euler characteristic $\chi(G)$ of $G$ is defined to be the integer

$$
\sum_{i=0}^{n}(-1)^{i} \operatorname{rank}_{\mathbb{Z}}\left(P_{i} \otimes_{\mathbb{Z} G} \mathbb{Z}\right) \text {. }
$$

If all the $P_{i}$ can be taken to be free $\mathbb{Z} G$-modules, then $G$ is said to be of type $F L$. One motivation for proving Theorem 2 was to use the techniques of [20] to derive the following result.

THEOREM 3. Let $H$ be a normal elementary amenable subgroup of the group $G$. If $G$ is of type $F L$ and $H \neq 1$, then $\chi(G)=0$.

In [20] Rosset proved Theorem 3 in the special case when $H$ is abelian. To establish the general version of Theorem 3 , we make the following two modifications to Rosset's paper. Firstly we require (2.3) and (3.1) of [20] to remain valid when $A$ is elementary amenable. This means that we need $\mathbb{C} A$ to be an Ore domain $[15,2.1 .14]$ when $A$ is elementary amenable, which is true by Theorem 1.3 of [13]. Secondly in (3.4) of [20], we want to allow $A$ to be elementary amenable. This step is also valid, by Theorem 2 .

However, there is now a simpler method to prove Theorem 3. In an earlier version of this paper, I posed the problem of giving an example of a group $G$ satisfying the hypotheses of Theorem 3 which does not have a nontrivial normal abelian subgroup. This has now been shown in [10] to have a negative answer, where it will be proved that a nontrivial elementary amenable group of finite cohomological dimension has a nontrivial characteristic abelian subgroup. The proof depends on extending the notion of Hirsch length from polycyclic groups to elementary amenable groups, which was worked out by Hillman in [9]. Thus if $G$ is as in Theorem 3, then $G$ has a nontrivial normal abelian subgroup and so Theorem 3 follows immediately from the special case when $H$ is abelian.

It should be noted that Cheeger and Gromov have proved an enhanced Theorem 3 with "elementary amenable" weakened to "amenable" [4]. Their methods are different from, but related to, the above methods. However, it is still an open problem as to whether 
there exists an amenable group of type $F P$ which is not elementary amenable. Also in [9], Hillman has used Theorem 2 to obtain results on 2-complexes and 4-manifolds.

To describe our results further, we need to establish some notation. Let $\mathscr{L}$ denote the set of bounded linear operators considered as acting on the left of $L^{2}(G)$. For $\alpha \in \mathbb{C} G$, we have a bounded linear map defined by $\beta \mapsto \alpha \beta$ (multiplication by $\alpha$ ) for all $\beta \in L^{2}(G)$; thus $\mathbb{C} G$ can be identified as a subring of $\mathscr{L}$. More generally for $n \in \mathbb{P}$, the ring of operators on $L^{2}(G)^{n}$ is $M_{n}(\mathscr{L})$, and $M_{n}(\mathbb{C} G)$ can be identified as a subring of $M_{n}(\mathscr{L})$. A nonzero divisor in a ring $R$ is an element $s$ such that $s r \neq 0 \neq r s$ for all $r \in R \backslash 0$. If $G$ is a torsion free elementary amenable group, then all nonzero elements of $\mathbb{C} G$ are nonzero divisors by Theorem 1.4 of [13]. Thus the following is a generalization of Theorem 2 .

TheOREM 4. Let $G$ be an elementary amenable group, let $n \in \mathbb{P}$ and let $\alpha \in M_{n}(\mathbb{C} G)$ be a nonzero divisor. Suppose the finite subgroups of $G$ have bounded order. If $0 \neq \beta \in L^{2}(G)^{n}$, then $\alpha \beta \neq 0$.

We now want to discuss $W^{*}$-algebras. Let $L^{\infty}(G)$ denote the set of all formal sums $\sum_{g \in G} a_{g} g \quad\left(a_{g} \in \mathbb{C}\right)$ such that $\sup _{g \in G}\left|a_{g}\right|<\infty$. Then $\mathbb{C} G \subseteq L^{2}(G) \subseteq L^{\infty}(G)$ and if $\alpha=\sum_{g \in G} a_{g} g, \beta=\sum_{g \in G} b_{g} g \in$ $L^{2}(G)$, we set

$$
\alpha \beta=\sum_{g, h \in G} a_{g} b_{h} g h
$$

which yields a multiplication $L^{2}(G) \times L^{2}(G) \rightarrow L^{\infty}(G)$. We also have an involution ${ }^{*}: L^{\infty}(G) \rightarrow L^{\infty}(G)$ defined by $\left(\sum a_{g} g\right)^{*}=\sum \bar{a}_{g} g^{-1}$, where - denotes complex conjugation, satisfying $L^{2}(G)^{*}=L^{2}(G)$ and $\mathbb{C} G^{*}=\mathbb{C} G$. By definition $W(G)$ is the weak closure of $\mathbb{C} G$ in $\mathscr{L}$; equivalently $W(G)$ is the $W^{*}$-algebra generated by $\mathbb{C} G$ in $\mathscr{L}$. Another description of $W(G)$ is that it is the double commutant $\mathbb{C} G^{\prime \prime}$ of $\mathbb{C} G$ in $\mathscr{L}$, where for any subset $S$ of $\mathscr{L}$, its commutant $\{\theta \in \mathscr{L} \mid \theta s=s \theta$ for all $s \in S\}$ is denoted by $S^{\prime}$ [1, Theorem 1.2.1]. We have a $*$-monomorphism $W(G) \rightarrow L^{2}(G)$ defined by $\theta \mapsto \theta(1)$. Thus $W(G)$ can be identified with a subspace of $L^{2}(G)$, where the action of $W(G)$ on $L^{2}(G)$ is left multiplication.

Since $M_{n}(W(G))$ is the double commutant of $M_{n}(\mathbb{C} G)$ in $M_{n}(\mathscr{L})$, we see that $M_{n}(W(G))$ is also a $W^{*}$-algebra [1, Theorem 1.2.1]. Let $\theta \in M_{n}(W(G))$ be represented by the matrix $\left(\theta_{i j}\right)$. Then $\theta^{*}$ is represented by the matrix $\left(\theta_{j i}^{*}\right)$. Furthermore right multiplication makes 
$L^{2}(G)^{n}$ into a right $\mathbb{C} G$-module, and $\theta$ is obviously a continuous right $\mathbb{C} G$-map. Conversely we have

LEMMA 5. If $\theta: L^{2}(G)^{n} \rightarrow L^{2}(G)^{n}$ is a continuous right $\mathbb{C} G$-map, then $\theta \in M_{n}(W(G))$.

Proof. This follows from Theorem 1 of Part 1, Chapter 5 of [7]; alternatively we give the following direct argument. Certainly $\theta \in$ $M_{n}(\mathscr{L})$ because $\theta$ is a bounded linear map, so write $\theta$ as a matrix with entries $\theta_{i j}(1 \leq i, j \leq n)$. Then $\theta_{i j}: L^{2}(G) \rightarrow L^{2}(G)$ is a continuous right $\mathbb{C} G$-map and it will be sufficient to prove that $\theta_{i j} \in$ $W(G)$; in other words we may assume that $n=1$.

Write $\theta(1)=\sum_{g \in G} a_{g} g \in L^{2}(G)$ where $a_{g} \in \mathbb{C}$. Since $\theta$ is a right $\mathbb{C} G$-map, $\theta(\alpha)=\theta(1) \alpha$ for all $\alpha \in \mathbb{C} G$. But $\theta$ is continuous and $\mathbb{C} G$ is dense in $L^{2}(G)$, so $\theta$ is left multiplication by $\theta(1)$. Suppose $\varphi \in \mathbb{C} G^{\prime}$ so that $g \varphi=\varphi g$ for all $g \in G$. Then for $h \in G$,

$$
\begin{aligned}
\varphi \theta(h)=\varphi(\theta(1) h) & =\varphi\left(\sum a_{g} g h\right) \\
& =\sum a_{g} \varphi(g h) \quad \text { (because } \varphi \text { is continuous) } \\
& =\sum a_{g} g \varphi(h)=\theta(1) \varphi(h)=\theta \varphi(h) .
\end{aligned}
$$

Since $\varphi \theta, \theta \varphi \in \mathscr{L}$ and $\mathbb{C} G$ is dense in $L^{2}(G)$, we deduce that $\varphi \theta=\theta \varphi$. Therefore $\theta \in \mathbb{C} G^{\prime \prime}$ so by the double commutant theorem $[1$, Theorem 1.2.1], we deduce that $\theta \in W(G)$ as required.

If $\alpha=\sum a_{g} g \in W(G)$, then we define $\operatorname{tr} \alpha=a_{1}$, and if $\theta \in$ $M_{n}(W(G))$ is represented by the matrix $\left(\theta_{i j}\right)$, then we define $\operatorname{Tr} \theta=$ $\sum_{i=1}^{n} \operatorname{tr} \theta_{i i}$. Note that $\operatorname{Tr} \theta \varphi=\operatorname{Tr} \varphi \theta$ for all $\theta, \varphi \in M_{n}(W(G))$. Following [6], an $L^{2}(G)$-module $M$ is a closed right $\mathbb{C} G$-submodule of $L^{2}(G)^{n}$ for some $n \in \mathbb{P}$, and a homomorphism of $L^{2}(G)$-modules is a continuous right $\mathbb{C} G$-map. Let

$$
e: L^{2}(G)^{n} \rightarrow L^{2}(G)^{n}
$$

be the projection onto $M$. Then $e$ is a continuous right $\mathbb{C} G$-map; thus $e \in M_{n}(W(G))$ by Lemma 5 and we define $d_{G} M=\operatorname{Tr} e$. Propositions 3 and 4 of [6] show that $d_{G} M$ is a nonnegative real number, and does not depend on the isomorphism type of $M$ (or $n$ ). If $\varphi \in M_{n}(\mathscr{L})$ is a continuous right $\mathbb{C} G$-map, then the closure of the subspace $\varphi\left(L^{2}(G)^{n}\right)$ is an $L^{2}(G)$-module, so by the above we can define $\operatorname{rank} \varphi=\operatorname{Tr} f$, where $f$ is the projection onto the closure of $\varphi\left(L^{2}(G)^{n}\right)$. If $\psi$ is a right $\mathbb{C} G$-endomorphism of $\mathbb{C} G^{n}$, then $\psi$ induces a continuous right 
$\mathbb{C} G$-endomorphism $\hat{\psi}$ of $L^{2}(G)^{n}$, and hence we can define rank $\psi=$ rank $\hat{\psi}$. As in [6], we let $D(G)$ indicate the additive subgroup of $\mathbb{R}$ generated by

$$
\left\{\operatorname{rank} \theta \mid \theta \in \operatorname{End}_{\mathbb{C} G}\left(\mathbb{C} G^{n}\right) \text { for some } n \in \mathbb{P}\right\} .
$$

The following theorem is a variant of the Goldie rank conjecture [19, $13]$ and extends the results of $[6, \S 5]$.

THEOREM 6. Let $G$ be an elementary amenable group and assume that the finite subgroups of $G$ have bounded order. If $l$ is the $l \mathrm{~cm}$ of the orders of the finite subgroups of $G$, then $D(G)=(1 / l) \mathbb{Z}$.

We now consider quotient rings. Let $R$ be a ring and let $S$ be the set of nonzero divisors of $R$. A classical quotient ring for $R$ is a ring $Q$ which contains $R$ as a subring such that every element of $S$ is invertible in $Q$, and

$$
Q=\left\{r s^{-1} \mid r \in R, s \in S\right\}=\left\{s^{-1} r \mid r \in R, s \in S\right\} .
$$

When a classical quotient ring exists, it is unique up to isomorphism. Recall that a ring $T$ is regular if for each $r \in T$, there exists $t \in T$ such that $r t r=r$. A regular ring is its own classical quotient ring, and a semisimple Artinian ring is a regular ring. If $R$ has a classical quotient ring $Q$ which is regular and $n \in \mathbb{P}$, then $M_{n}(Q)$ is a regular classical quotient ring for $M_{n}(R)$ (use Exercise $12 \mathrm{C}$ on p. 232 of [2]). Since $W(G)$ is a finite $W^{*}$-algebra by a well-known result of Kaplansky (see [14, Proposition 9]), the results of [3] (in particular the proof of Theorem 10) show that $W(G)$ has a regular classical quotient ring; hence $M_{n}(W(G))$ has a regular classical quotient ring $U$. Suppose in addition that $G$ is elementary amenable and that its finite subgroups have bounded order. Applying [13, §5] and [8, Exercise 3.D.9], we see that $M_{n}(\mathbb{C} G)$ has a classical quotient ring $Q$, which is a semisimple Artinian ring. By Theorem 4 every nonzero divisor of $M_{n}(\mathbb{C} G)$ is a nonzero divisor in $M_{n}(W(G))$ and hence is invertible in $U$. Thus the inclusion map $M_{n}(\mathbb{C} G) \rightarrow M_{n}(W(G))$ extends uniquely to a monomorphism $Q \rightarrow U$ [15, Lemma 2.1.4] so we can view $Q$ as a subring of $U$. Moreover the involution * of $M_{n}(W(G))$ extends uniquely to an involution of $U$ satisfying $Q=Q^{*}$ [3, 8, Exercise 2.A.17]. Summing up this paragraph we have

THEOREM 7. Let $G$ be an elementary amenable group, let $n \in \mathbb{P}$, and assume that the finite subgroups of $G$ have bounded order. Then 
$M_{n}(\mathbb{C} G)$ and $M_{n}(W(G))$ have classical quotient rings $Q$ and $U$ respectively such that $Q \subseteq U$. Furthermore $Q$ is a semisimple Artinian ring, $U$ is a regular ring, and the involution * on $M_{n}(W(G))$ extends to an involution (also denoted ${ }^{*}$ ) on $U$ satisfying $Q=Q^{*}$.

As expected, this paper relies heavily on the affirmative answer to the zero divisor conjecture for elementary amenable groups. This was recorded in Theorem 1.4 of [13], and was made possible by Moody's remarkable induction theorem [16]. We give a brief outline of the proof of Theorem 2 to illustrate the techniques employed in this paper. Using the description of elementary amenable groups in $\S 3$ of [13], a transfinite induction argument reduces to considering the case when there is a normal subgroup $H$ of $G$ such that $G / H$ is either finite or infinite cyclic, and the theorem is true with $H$ in place of $G$. If $G / H$ is finite, then the solution to the zero divisor conjecture implies that there exists $\gamma \in \mathbb{C} G$ such that $\gamma \alpha \in \mathbb{C} H \backslash 0$, and the result follows in this case. If $G / H$ is infinite cyclic, we use the methods of [21]. Write $G=\langle H, x\rangle$ and let $\zeta \in \mathbb{C}$ with $|\zeta|=1$. What will be important here is that the map $h \mapsto h, x \mapsto x \zeta$ for $h \in H$ induces an automorphism of $\mathbb{C} G$ which extends to an automorphism of $W(G)$.

I would like to thank Dan Farkas for some useful comments on a preliminary version of this paper.

2. Notation, terminology, and assumed results. All rings will be associative with a 1 , and subrings will have the same 1 . As usual $\mathbb{P}$ will denote the positive integers $\{1,2, \ldots\}$ and if $\theta$ is a map, then $\operatorname{ker} \theta$ will denote the kernel of $\theta$. Suppose $R$ is a ring, $n \in \mathbb{P}$ and $S \subseteq R$. Then $S^{\prime}$ will denote the commutant

$$
\{r \in R \mid r s=s r \text { for all } s \in S\}
$$

of $S$ in $R, R^{n}$ the right $R$-module which is the direct sum of $n$ copies of $R, M_{n}(R)$ the ring of $n \times n$ matrices with entries in $R$, which we shall also view as the $R$-endomorphisms of $R^{n}$ where the action is left multiplication, $\mathrm{GL}_{n}(R)$ the invertible elements of $M_{n}(R)$, $1_{n}$ the identity matrix of $M_{n}(R)$, and $0_{n}$ the zero matrix of $M_{n}(R)$. If $t \in \mathbb{P}$ and $A_{i} \in M_{n_{t}}(R) \quad(i=1,2, \ldots, t)$, then $\operatorname{diag}\left(A_{1}, \ldots, A_{t}\right)$ denotes the matrix in $M_{n_{1}+\cdots+n_{t}}(R)$

$$
\left(\begin{array}{cccc}
A_{1} & 0 & . & 0 \\
0 & A_{2} & \cdot & 0 \\
\dot{0} & \dot{0} & 0 & 0 \\
0 & 0 & A_{t}
\end{array}\right) .
$$


Suppose $P$ is a finitely generated projective right $R$-module. Then we can write $P \oplus P^{\prime}=R^{n}$ for some right $R$-module $P^{\prime}$ and some $n \in \mathbb{P}$. If $e \in M_{n}(R)$ is the idempotent satisfying $e(P)=P, e\left(P^{\prime}\right)=0$, then we shall say that $e$ corresponds to $P$. Suppose $Q$ is a right $R$ module such that $Q \simeq P$ and $Q \oplus Q^{\prime}=R^{m}$ for some right $R$-module $Q^{\prime}$ and some $m \in \mathbb{P}$. Let $f \in M_{m}(R)$ be the idempotent satisfying $f(Q)=Q, f\left(Q^{\prime}\right)=0$ so $f$ also corresponds to $P$. Then

$$
P^{\prime} \oplus R^{m} \simeq R^{n} \oplus Q^{\prime}
$$

hence there is an automorphism $\theta$ of $R^{n} \oplus R^{m}$ such that $\theta(P)=$ $Q$ and $\theta\left(P^{\prime} \oplus R^{m}\right)=R^{n} \oplus Q^{\prime}$. Consequently $\theta \operatorname{diag}\left(e, 0_{m}\right) \theta^{-1}=$ $\operatorname{diag}\left(0_{n}, f\right)$. We can now state

LeMma 8. Let $R$ be a ring, let $m, n \in \mathbb{P}$, and let $P, Q$ be finitely generated projective right $R$-modules such that $P \simeq Q$. If $P$ and $Q$ correspond to the idempotents $e \in M_{n}(R)$ and $f \in M_{m}(R)$ respectively, then there exists $u \in \mathrm{GL}_{n+m}(R)$ such that $u \operatorname{diag}\left(e, 0_{m}\right) u^{-1}=$ $\operatorname{diag}\left(f, 0_{n}\right)$.

If $H \leq G$ are groups, then $|H|$ denotes the order of $H, G: H$ the index of $H$ in $G$, and $\mathscr{F}(G)$ the set of finite subgroups of $G$.

LEMMA 9. Let $G$ be an elementary amenable group, let $H \triangleleft G$ with $G: H<\infty$, let $n \in \mathbb{P}$, and let $\alpha \in M_{n}(\mathbb{C} G)$ be a nonzero divisor. If the finite subgroups of $G$ have bounded order then there exists $\beta \in M_{n}(\mathbb{C} G)$ such that $\beta \alpha$ is a nonzero divisor in $M_{n}(\mathbb{C} H)$.

Proof. Let $S$ be the set of nonzero divisors in $M_{n}(\mathbb{C H})$. Then Theorem 1.2 of [13] shows that $M_{n}(\mathbb{C H})$ has an Artinian left quotient ring of the form $S^{-1} M_{n}(\mathbb{C} H) \quad\left(=\left\{s^{-1} \gamma \mid s \in S, \gamma \in M_{n}(\mathbb{C} H)\right\}\right)$; hence $S^{-1} M_{n}(\mathbb{C} G)$ is an Artinian left quotient ring for $M_{n}(\mathbb{C} G)$. This means that in $S^{-1} M_{n}(\mathbb{C} G)$, we can write $\alpha^{-1}=\sigma^{-1} \beta$ where $\sigma \in S$ and $\beta \in M_{n}(\mathbb{C} G)$; thus $\beta \alpha=\sigma$ as required.

LEMMA 10. Let $H \leq G$ be groups, let $n \in \mathbb{P}$, and let $\theta \in M_{n}(W(H))$. If $\theta \alpha=0$ for some $\alpha \in L^{2}(G)^{n} \backslash 0$, then $\theta \beta=0$ for some $\beta \in$ $L^{2}(H)^{n} \backslash 0$.

Proof. Let $T$ be a set of right coset representatives for $H$ in $G$. Then we can write $\alpha=\sum_{t \in T} \alpha_{t} t$ where $\alpha_{t} \in L^{2}(H)^{n}$, and there exists $s \in T$ such that $\alpha_{s} \neq 0$. Since $\theta \alpha=0$, we see that $\theta \alpha_{s}=0$, as required. 
Let $U$ be the classical quotient ring of $M_{n}(W(G))$ and let $T \subseteq U$. Since $U$ is a Baer *-ring (see $\S 3$ of [3]), there is a unique projection $e \in U$ such that

$$
\{u \in U \mid t u=0 \text { for all } t \in T\}=e U
$$

We shall write $N(T)=e, L P\left(T^{*}\right)=1-e$ (thus $L P(T)$ is the "left projection" of $T$ in $U$; cf. p. 13 of [2]), and $L P(\alpha)=L P(\{\alpha\})$ for $\alpha \in U$. We note that $e \in M_{n}(W(G))$ because every projection of $U$ lies in $M_{n}(W(G))$ (see [3]). Then we have

LEMMA 11. Let $G$ be a group, let $n \in \mathbb{P}$, let $U$ be the classical quotient ring of $M_{n}(W(G))$, let $T \subseteq M_{n}(W(G))$ and let $\alpha \in M_{n}(W(G))$.

(i) If $e$ is a projection such that $e U=\alpha U$, then $e=L P(\alpha)$.

(ii) The projection of $L^{2}(G)^{n}$ onto $\operatorname{ker} \alpha$ is $N(\alpha)$. In particular if $\alpha$ is a nonzero divisor in $M_{n}(W(G))$, then $\operatorname{ker} \alpha=0$.

(iii) Let $F$ be the closure of $\sum_{\theta \in T} \theta\left(L^{2}(G)^{n}\right)$. Then the projection of $L^{2}(G)^{n}$ onto $F$ is $L P(T)$.

Proof. For (i) and (ii), see p. 13 of [2]. Now let $f$ be the projection of $L^{2}(G)^{n}$ onto $F$, and let $h=L P(T)$. Then $f \in M_{n}(W(G))$ by Lemma 5 and $(1-f) T=0$; hence $T^{*}(1-f)=0$ and we see that $(1-f) U \subseteq(1-h) U$. Also $T^{*}(1-h)=0$. Thus $h t=t$ for all $t \in T$ and we deduce that $h\left(L^{2}(G)^{n}\right) \supseteq F$. Therefore $h f=f$, consequently $(1-h) U \subseteq(1-f) U$ and it follows that $(1-f) U=(1-h) U$. This yields $f=h$, as required.

LEMMA 12. Let $G$ be a group, let $n \in \mathbb{P}$, let $e, f$ be projections in $M_{n}(W(G))$ and let $h=L P(e, f)$. If $e M_{n}(W(G)) \cap f M_{n}(W(G))=0$, then $\operatorname{Tr} e+\operatorname{Tr} f=\operatorname{Tr} h$.

Proof. Let $L=L^{2}(G)^{n}$ and let $\pi$ be the projection onto $e L \cap f L$. Since $e L$ and $f L$ are $L^{2}(G)$-modules, $e L \cap f L$ is also an $L^{2}(G)$ module. Hence $\pi \in M_{n}(W(G))$ by Lemma 5 . Now $e \pi=\pi=f \pi$, thus $\pi \in e M_{n}(W(G)) \cap f M_{n}(W(G))=0$ and we deduce that $e L \cap$ $f L=0$.

Define $\theta: e L \oplus f L \rightarrow h L$ by $\theta(u, v)=u+v$. Though $\theta$ is not necessarily an isomorphism, it is certainly true that $\operatorname{ker} \theta=0$ and 
$\theta(e L \oplus f L)$ is dense in $h L$ (Lemma 11(iii)); in the terminology of [6, p. 133] $\theta$ is a weak isomorphism. Thus by von Neumann's theorem $\left([17, \S 21.1]\right.$ and [6, p. 134]) $e L \oplus f L \simeq h L$ as $L^{2}(G)$-modules; hence $\operatorname{Tr} e+\operatorname{Tr} f=\operatorname{Tr} h$.

We note that this result could also be fairly easily derived from the parallelogram law $[2, \S 13]$.

LEMMA 13. Let $G$ be a group, let $n \in \mathbb{P}$, let $U$ be the classical quotient ring of $M_{n}(W(G))$, and let $e, f$ be projections in $U$. If $f=\mathrm{ueu}^{-1}$ for some unit $u \in U$, then $f=v e v^{-1}$ for some unit $v \in M_{n}(W(G))$.

Proof. Clearly $e$ and $f$ are algebraically equivalent in $U$ (cf. Exercise $6 \mathrm{~A}$ on p. 8 of [2]). Using the theory of $\S 5$ of [3], we see that $e$ and $f$ are perspective in $M_{n}(W(G))$. Since $M_{n}(W(G))$ is a finite $W^{*}$-algebra by Kaplansky's Theorem [14, Proposition 9], it follows from Exercise $12 \mathrm{~A}$ (viii) on p. 109 of [2] that $e$ and $f$ are unitarily equivalent in $M_{n}(W(G))$; in particular $f=v e v^{-1}$ for some unit $v \in M_{n}(W(G))$.

A division $\mathbb{C}$-algebra is a division ring whose center contains $\mathbb{C}$.

LeMma 14. Let $D$ be a division $\mathbb{C}$-algebra and let $f(t) \in D[t]$. If $f(0) \neq 0$, then $f(z)=0$ for only finitely many $z \in \mathbb{C}$.

Proof. This is elementary.

LemMA 15. Let $D$ be a division $\mathbb{C}$-algebra, let $n \in \mathbb{P}$ and let $A(t) \in$ $M_{n}(D[t])$. If $A(0) \in M_{n}(D)$ is invertible, then $A(z)$ is invertible in $M_{n}(D)$ for all but finitely many $z \in \mathbb{C}$.

Proof. According to Theorem 16 on p. 43 of [11], there exist $X(t)$, $Y(t) \in \mathrm{GL}_{n}(D[t])$ such that $X(t) A(t) Y(t)$ is a diagonal matrix, say $\operatorname{diag}\left(f_{1}(t), \ldots, f_{n}(t)\right)$ for some $f_{i}(t) \in D[t]$. Since $A(0)$ is invertible, $f_{1}(0), \ldots, f_{n}(0)$ are all nonzero. Therefore $f_{1}(z), \ldots, f_{n}(z)$ are all nonzero for all but finitely many $z \in \mathbb{C}$ by Lemma 14 , and hence $A(z)$ is invertible for all but finitely many $z \in \mathbb{C}$, as required.

3. Proofs. Let $D$ be a ring and let $\sigma$ be an automorphism of $D$. Then we can form the skew polynomial ring $D[x, \sigma]$ whose elements 
consist of all finite formal sums $\sum_{i=0}^{n} a_{i} x^{i} \quad\left(a_{i} \in D\right)$ with multiplication defined by

$$
\left(\sum_{i=0}^{n} a_{i} x^{i}\right)\left(\sum_{j=0}^{m} b_{j} x^{j}\right)=\sum_{r=0}^{m+n}\left(\sum_{i+j=r} a_{i}\left(\sigma^{i} b_{j}\right)\right) x^{r}
$$

(cf. 2.1 of [15]). If $f(x)=\sum_{i=0}^{n} a_{i} x^{i} \in D[x, \sigma]$ and $p \in D[x, \sigma]$, then we set $f(p)=\sum_{i=0}^{n} a_{i} p^{i}$.

LEMMA 16. Let $D$ be a division $\mathbb{C}$-algebra, let $\sigma$ be an automorphism of $D$, and let $f(x) \in D[x, \sigma]$. If $f(0)=1$, then for all but finitely many $t \in \mathbb{C}$,

$$
D[x, \sigma] f(x)+D[x, \sigma] f(t x)=D[x, \sigma] .
$$

Proof. Extend $\sigma$ to an automorphism of the polynomial ring $E=$ $D[y]$ by setting $\sigma(y)=y$, and write $f(x)=1+a_{1} x+\cdots+a_{n} x^{n}$ where $a_{i} \in D$ and $a_{n} \neq 0$. Then $f(y x)=1+a_{1} y x+\cdots+a_{n} y^{n} x^{n} \in$ $E[x, \sigma]$. We want to show that for all but finitely many $t \in \mathbb{C}$, there exist $g(x)=b_{1} x+\cdots+b_{n} x^{n}$ and $h(x)=1+c_{1} x+\cdots+c_{n} x^{n}$ $\left(b_{i}, c_{i} \in D\right)$ such that $g(x) f(x)+h(x) f(t x)=1$ ( $g$ and $h$ depending on $t)$. We consider the equation $g(x) f(x)+h(x) f(y x)=1$ and equate coefficients to obtain $2 n$ linear equations in the $2 n$ unknowns $b_{1}, \ldots, b_{n}, c_{1}, \ldots, c_{n}$ with coefficients in $D[y]$ :

(1) $b_{1}+c_{1}=-a_{1} y$,

$$
\begin{aligned}
& b_{1}\left(\sigma a_{1}\right)+b_{2}+c_{1}\left(\sigma a_{1}\right) y+c_{2}=-a_{2} y^{2}, \\
& b_{1}\left(\sigma a_{2}\right)+b_{2}\left(\sigma^{2} a_{1}\right)+b_{3}+c_{1}\left(\sigma a_{2}\right) y^{2}+c_{2}\left(\sigma^{2} a_{1}\right) y+c_{3}=-a_{3} y^{3}, \\
& \quad \ldots \\
& \quad b_{n-1}\left(\sigma^{n-1} a_{n}\right)+b_{n}\left(\sigma^{n} a_{n-1}\right) \\
& \quad+c_{n-1}\left(\sigma^{n-1} a_{n}\right) y^{n}+c_{n}\left(\sigma^{n} a_{n-1}\right) y^{n-1}=0, \\
& b_{n}\left(\sigma^{n} a_{n}\right)+c_{n}\left(\sigma^{n} a_{n}\right) y^{n}=0 .
\end{aligned}
$$

Let $A(y) \in M_{2 n}(D[y])$ be the matrix of coefficients of (1). We note that when $y=0$, the above system has a unique solution, namely $b_{i}=c_{i}=0$ for all $i$. Therefore $A(0) \in \mathrm{GL}_{2 n}(D)$, and hence $A(t) \in$ $\mathrm{GL}_{2 n}(D)$ for all but finitely many $t \in \mathbb{C}$ by Lemma 15 . Thus (1) has a unique solution when $y=t$ for all but finitely many $t \in \mathbb{C}$, and the result follows.

Proof of Theorem 4. We shall prove the result by transfinite induction, using the description of elementary amenable groups of $\S 3$ of 
[13]; we shall also adopt the notation of that section. Thus if $\mathscr{Y}$ and $\mathscr{Z}$ are classes of groups, then $H \in L \mathscr{Y}$ means that every finite subset of the group $H$ is contained in a $\mathscr{Y}$-subgroup, $H \in \mathscr{Y} \mathscr{Z}$ means that $H$ has a normal $\mathscr{Y}$-subgroup $Y$ such that $H / Y \in \mathscr{Z}$, and $\mathscr{B}$ denotes the class of finitely generated abelian by finite groups. Also for each ordinal $a$, the class of groups $\mathscr{Z}_{a}$ is defined inductively by $\mathscr{X}_{0}=\{1\}, \mathscr{X}_{a+1}=\left(L \mathscr{X}_{a}\right) \mathscr{B}$ and $\mathscr{X}_{b}=\bigcup_{a<b} \mathscr{Z}_{a}$ if $b$ is a limit ordinal. Following $\S 3$ of [13], choose the least ordinal $a$ such that $G \in \mathscr{X}_{a}$, and assume that the result is true whenever $G \in \mathscr{Z}_{b}$ and $b<a$.

Now $a$ cannot be a limit ordinal, and the result is clearly true if $a=0$. Therefore we may assume that $a=c+1$ for some ordinal $c$. First suppose $G \in L \mathscr{Z}_{c}$. Then $\alpha \in M_{n}(\mathbb{C} A)$ for some $A \in \mathscr{X}_{c}$. Hence $\alpha \beta \neq 0$ by induction and Lemma 10.

Now suppose there exists $H \triangleleft G$ such that $G / H \in \mathscr{B}$ and $H \in$ $L \mathscr{X}_{c}$. Let $B / H$ be a torsion free abelian normal subgroup of finite index in $G / H$. By Lemma 9 there exists $\gamma \in M_{n}(\mathbb{C} G)$ such that $\gamma \alpha$ is a nonzero divisor in $M_{n}(\mathbb{C} B)$. If the result is true for $B$, then $\gamma \alpha \beta \neq 0$ by Lemma 10 ; hence $\alpha \beta \neq 0$ as required. In other words we may assume that $G / H$ is finitely generated free abelian. A simple induction argument on the rank of $G / H$ allows us to assume that $G / H$ is infinite cyclic, say $G=H\langle x\rangle$ for some $x \in G$.

Let $Q$ and $U$ denote the classical quotient rings of $M_{n}(\mathbb{C} H)$ and $M_{n}((W(H))$ respectively. Since the result is true for $H$, every nonzero divisor in $M_{n}(\mathbb{C} H)$ is a nonzero divisor in $M_{n}(W(H))$. Thus we may view $Q$ as a subring of $U$ (cf. Theorem 7). Now $Q$ is a semisimple Artinian ring (cf. Theorem 7), so we may write

$$
Q=\bigoplus_{i=1}^{t} M_{n_{i}}\left(D_{i}\right)
$$

for some $n_{i} \in \mathbb{P}$ and division rings $D_{i}$. Let $D=\bigoplus_{i=1}^{t} D_{i}$ and note that the map $v \mapsto x^{-1} v x$ for $v \in Q$ is an automorphism of $Q$. Since an automorphism of $Q$ permutes the $M_{n_{i}}\left(D_{i}\right)$, there exists $r \in \mathbb{P}$ such that $x^{-r} M_{n_{i}}\left(D_{i}\right) x^{r}=M_{n_{i}}\left(D_{i}\right)$ for all $i$. If the result is true for $H\left\langle x^{r}\right\rangle$, then the result is true for $H\langle x\rangle$ by the argument of the previous paragraph. Therefore we may assume that $r=1$, in other words $x^{-1} M_{n_{i}}\left(D_{i}\right) x=M_{n_{i}}\left(D_{i}\right)$ for all $i$. The corollary on p. 237 of [12] applied to each $M_{n_{i}}\left(D_{i}\right)$ shows that there exists an automorphism $\theta$ of $D$ which fixes the $D_{i}$ setwise, and a unit $u \in Q$ such that $x^{-1} v x=u^{-1}\left(\theta_{*} v\right) u$ for all $v \in Q$, where $\theta_{*}$ is the automorphism of $Q$ induced by $\theta$. If $y=u x^{-1}$, then $Q\left[y, y^{-1}\right]=Q\left[x, x^{-1}\right]$ and $y D_{i} y^{-1}=D_{i}$ for all $i$. 
Let $V$ be the classical quotient ring of $M_{n}(W(G))$ and let $S=\sup \left\{\operatorname{Tr}(N(f)) \mid f\right.$ is a nonzero divisor in $\left.D\left[y, y^{-1}\right]\right\}$.

We claim that every nonzero divisor in $D\left[y, y^{-1}\right]$ is a nonzero divisor in $V$. If this is false, then $0<S \leq n$ by Kaplansky's theorem [14, Proposition 9], so we can choose a nonzero divisor $f$ in $D\left[y, y^{-1}\right]$ such that $\operatorname{Tr}(N(f))>S / 2$. Write $f=f_{1}+\cdots+f_{t}$ where $f_{i} \in$ $D_{i}\left[y, y^{-1}\right]$. Note that $f_{i} \neq 0$ for all $i$ because $f$ is a nonzero divisor. Now $D_{i}[y]$ is a skew polynomial ring, thus applying Lemma 16 for $i=1,2, \ldots, t$, we infer that there exists $\zeta \in \mathbb{C},|\zeta|=1$, such that

$$
D\left[y, y^{-1}\right] f(y)+D\left[y, y^{-1}\right] f(\zeta y)=D\left[y, y^{-1}\right] .
$$

Let $e=N(f)$ and let $\varphi$ be the automorphism of $V$ induced by $h \mapsto$ $h, x \mapsto x \bar{\zeta}$ for all $h \in H$. Note that $\varphi(f(y))=f(\zeta y), N(\varphi(f))=$ $\varphi(e)$ and $\operatorname{Tr} \varphi(e)=\operatorname{Tr} e>S / 2$. Using (2) we deduce that $e V \cap$ $\varphi(e) V=0$, hence by Lemma 12

$$
\operatorname{Tr} L P(e, \varphi(e))=\operatorname{Tr} e+\operatorname{Tr} \varphi(e)>S .
$$

Therefore if $f_{0} \in D\left[y, y^{-1}\right] f(y) \cap D\left[y, y^{-1}\right] f(\zeta y)$, then since $f_{0} L P(e, \varphi(e))=0$, we see that $\operatorname{Tr} N\left(f_{0}\right)>S$. But $D\left[y, y^{-1}\right] f(y)$ $\cap D\left[y, y^{-1}\right] f(\zeta y)$ contains nonzero divisors in $D\left[y, y^{-1}\right]$ and the claim is established. It follows that every nonzero divisor in $D\left[y, y^{-1}\right]$ is invertible in $V$.

Let $e_{i j}^{k}\left(1 \leq i, j \leq n_{k}, 1 \leq k \leq t\right)$ be the standard matrix units of $Q$, let $e^{k}=\sum_{i} e_{i i}^{k}$ be the primitive central idempotents of $Q$, and let

$$
C=\left\{c d^{-1} \mid c, d \in D\left[y, y^{-1}\right] \text { and } d\right. \text { is a nonzero divisor }
$$

$$
\text { in } \left.D\left[y, y^{-1}\right]\right\} \text {. }
$$

Notice that each $D_{k}\left[y, y^{-1}\right]$ has a classical quotient ring which is a division ring; thus each $C e^{k}$ is a division ring $(1.2 .9,2.1$ of [15]). Since $C e^{k}$ centralizes all the $e_{i j}^{k}$, it follows that

$$
\sum_{i, j, k} C e_{i j}^{k} \simeq \bigoplus_{k} M_{n_{k}}\left(C e^{k}\right),
$$

a subring of $V$ in which nonzero divisors are invertible. By construction $M_{n}(\mathbb{C} G)$ is a subring of $\sum_{i, j, k} C e_{i j}^{k}$, and a nonzero divisor in $M_{n}(\mathbb{C} G)$ remains a nonzero divisor in $\sum_{i, j, k} C e_{i j}^{k}$. Therefore $\alpha$ is invertible in $V$; hence $\alpha$ is a nonzero divisor in $M_{n}(W(G))$ and the result follows from Lemma 11(ii). 
For the proof of Theorem 6, we need the following technical result, which depends ultimately on Moody's induction theorem [16].

LEMMA 17. Suppose $G$ is an elementary amenable group whose $f$ nite subgroups have bounded order. Let $Q$ be the classical quotient ring of $\mathbb{C} G$, let $n \in \mathbb{P}$, and let $e \in M_{n}(Q)$ be an idempotent. Then there exist $r, s \in \mathbb{P}$, finite subgroups $F_{1}, \ldots, F_{s}$ of $G$ and projections $f_{i} \in \mathbb{C} F_{i}(1 \leq i \leq s)$ such that

$$
\operatorname{diag}\left(e, 1_{r}, 0_{s}\right)=u \operatorname{diag}\left(f_{1}, \ldots, f_{s}, 0_{n+r}\right) u^{-1}
$$

where $u \in \mathrm{GL}_{n+r+s}(Q)$.

Proof. Write $N=\Delta^{+}(G)$, the torsion subgroup of the finite conjugate subgroup of $G$ (see p. 2 of [18]). We shall apply the theory of $\S 5$ of [13]. Write $\mathbb{C} N=R_{1} \oplus \ldots \oplus R_{m}$ where the $R_{i}$ are matrix rings over $\mathbb{C}$. Then $G / N$ permutes the $R_{i}$ by conjugation and by renumbering if necessary, we may assume that $\left\{R_{1}, \ldots, R_{t}\right\}$ is a set of orbit representatives for this action. Let $G_{i} / N$ be the stabilizer of $R_{i}$, and write $n_{i}=G: G_{i}$. By Clifford's theorem

$$
\mathbb{C} G=\mathbb{C} N * G / N=\bigoplus_{i=1}^{t} M_{n_{i}}\left(R_{i} * G_{i} / N\right) .
$$

Let $Q_{i}$ be the simple Artinian quotient ring of $M_{n_{i}}\left(R_{i} * G_{i} / N\right)=$ $M_{n_{i}}\left(R_{i}\right) * G_{i} / N \quad(1 \leq i \leq t)$, which exists by Lemma 4.1(i) of [13]. Then $Q=\bigoplus_{i=1}^{t} Q_{i}$. Using Lemma 4.1(ii) of [13], we see that the natural induction map

$$
\bigoplus_{F \in \mathscr{F}\left(G_{i}\right)} G_{0}\left(M_{n_{i}}\left(R_{i}\right) * F N / N\right) \rightarrow G_{0}\left(Q_{i}\right)
$$

is onto $(1 \leq i \leq t)$, and we can now infer that the natural induction map

$$
\bigoplus_{F \in \mathscr{F}(G)} G_{0}(\mathbb{C} F) \rightarrow G_{0}(Q)
$$

is also onto. Furthermore, all $Q$-modules are projective. This means that if $P$ is the projective $Q$-module corresponding to $e$, then there exist $r, s \in \mathbb{P}$, finite subgroups $F_{1}, \ldots, F_{s}$ of $G$ and finitely generated $\mathbb{C} F_{i}$-modules $P_{i} \quad(1 \leq i \leq s)$ such that

$$
P \oplus Q^{r} \simeq \bigoplus_{i=1}^{s} P_{i} \otimes_{\mathbb{C} F_{l}} Q \quad \text { (use }[15,12.1 .4] \text { ). }
$$


Since a finitely generated $\mathbb{C} F_{i}$-module is isomorphic to a direct sum of right ideals of $\mathbb{C} F_{i}$, we may assume that $P_{i} \simeq f_{i} \mathbb{C} F_{i}$ for some projection $f_{i}(1 \leq i \leq s)$. Then $\operatorname{diag}\left(e, 1_{r}\right)\left(Q^{n+r}\right) \simeq \operatorname{diag}\left(f_{1}, \ldots, f_{s}\right)\left(Q^{s}\right)$ as $Q$-modules; hence by Lemma 8

$$
\operatorname{diag}\left(e, 1_{r}, 0_{s}\right)=u \operatorname{diag}\left(f_{1}, \ldots, f_{s}, 0_{n+r}\right) u^{-1}
$$

for some $u \in \mathrm{GL}_{n+r+s}(Q)$, as required.

Proof of Theorem 6. Suppose $H$ is a finite subgroup of $G$. Then

$$
e=\frac{1}{|H|} \sum_{h \in H} h
$$

is a projection so if $\varepsilon: \mathbb{C} G \rightarrow \mathbb{C} G$ denotes left multiplication by $e$, then $\operatorname{rank} \varepsilon=1 /|H|$. Thus certainly $\langle 1 / l\rangle \subseteq D(G)$.

Conversely suppose $\theta: \mathbb{C} G^{n} \rightarrow \mathbb{C} G^{n}$ is a right $\mathbb{C} G$-map; equivalently $\theta \in M_{n}(\mathbb{C} G)$. Let $Q$ and $U$ denote the classical quotient rings of $\mathbb{C} G$ and $W(G)$ respectively, and assume that $Q \subseteq U$ (use Theorem 7). Since $M_{n}(Q)$ is a *-regular ring, there is a projection $e \in M_{n}(Q)$ such that $\theta M_{n}(Q)=e M_{n}(Q)$. Then $\theta M_{n}(U)=e M_{n}(U)$ and $e \in$ $M_{n}(W(G))$, hence $\operatorname{rank} \theta=\operatorname{Tr} e$ by Lemma 11. Applying Lemma 17, there exist $r, s \in \mathbb{P}$, finite subgroups $F_{1}, \ldots, F_{s}$ of $G$ and projections $f_{i} \in \mathbb{C} F_{i} \quad(1 \leq i \leq s)$ such that

$$
\operatorname{diag}\left(e, 1_{r}, 0_{s}\right)=u \operatorname{diag}\left(f_{1}, \ldots, f_{s}, 0_{n+r}\right) u^{-1}
$$

where $u \in \mathrm{GL}_{n+r+s}(Q)$. In view of Lemma 13, we may assume that $u \in \mathrm{GL}_{n+r+s}(W(G))$, hence

$$
\operatorname{Tr} e+r=\operatorname{Tr} f_{1}+\cdots+\operatorname{Tr} f_{s} .
$$

But $\operatorname{Tr} f_{i}=1 /\left|F_{i}\right| \in\langle 1 / l\rangle$ for all $i$, and the result follows.

\section{REFERENCES}

[1] W. Arveson, An Invitation to $C^{*}$-Algebra, Graduate Texts in Math., no. 3, Springer-Verlag, Berlin-New York, 1976.

[2] S. K. Berberian, Baer *-rings, Grundlehren, Band 195, Springer-Verlag, BerlinNew York, 1972.

[3] _ The maximal ring of quotients of a finite von Neumann algebra, Rocky Mountain J. Math., 12 (1982), 149-164.

[4] J. Cheeger and M. Gromov, $L_{2}$-cohomology and group cohomology, Topology, 25 (1986), 189-215.

[5] C. Chou, Elementary amenable groups, Illinois J. Math., 24 (1980), 396-407. 
[6] J. M. Cohen, Von Neumann dimension and the homology of covering spaces, Quart. J. Math. Oxford, 30 (1979), 133-142.

[7] J. Dixmier, Von Neumann Algebras, North-Holland, Amsterdam-New York, 1981.

[8] K. R. Goodearl, Ring Theory: Nonsingular Rings and Modules, Marcel Dekker, New York, 1976.

[9] J. A. Hillman, Elementary amenable groups and 4-manifolds with Euler characteristic 0, J. Austral. Math. Soc., to appear.

[10] J. A. Hillman and P. A. Linnell, Elementary amenable groups of finite Hirsch length are locally-finite by virtually solvable, J. Austral. Math. Soc., to appear.

[11] N. Jacobson, The Theory of Rings, Math. Surveys vol. II, Amer. Math. Soc., Providence, R.I., 1943.

[12] __ Lectures in Abstract Algebra II Linear Algebra, Graduate Texts in Math., no. 31, Springer-Verlag, Berlin-New York, 1975.

[13] P. H. Kropholler, P. A. Linnell and J. A. Moody, Applications of a new Ktheoretic theorem to soluble group rings, Proc. Amer. Math. Soc., 104 (1988), 675-684.

[14] P. A. Linnell, On accessibility of groups, J. Pure Appl. Algebra, 30 (1983), 39-46.

[15] J. C. McConnell and J. C. Robson, Noncommutative Noetherian Rings, WileyInterscience, New York, 1987.

[16] J. A. Moody, Brauer induction for $G_{0}$ of certain infinite groups, J. Algebra, 122 (1989), 1-14.

[17] M. A. Naimark, Normed Algebras, Wolters-Noordhoff Publishing, Groningen, 1972.

[18] D. S. Passman, Group Rings, Crossed Products and Galois Theory, CBMS Regional Conf. Ser. in Math., no. 64, Amer. Math. Soc., Providence, R. I., 1986.

[19] S. Rosset, The Goldie rank of virtually polycyclic groups, The Brauer Group, LNM 844, Springer-Verlag, Berlin-New York, 1981, pp. 35-45.

[20] _ A vanishing theorem for Euler characteristics, Math. Z., 185 (1984), 211215.

[21] M. K. Smith, Central zero divisors in group algebras, Proc. Amer. Math. Soc., 91(1984), 529-531.

Received November 16, 1989.

Virginia Polytechnic Institute and State University

BLACKSBURG, VA 24061-0123 



\section{PACIFIC JOURNAL OF MATHEMATICS EDITORS}

\author{
V. S. VARAdaraJan \\ (Managing Editor) \\ University of California \\ Los Angeles, CA 90024-1555-05 \\ Herbert Clemens \\ University of Utah \\ Salt Lake City, UT 84112 \\ THOMAS ENRIGHT \\ University of California, San Diego \\ La Jolla, CA 92093
}

R. FINN

Stanford University

Stanford, CA 94305

Hermann FlaschKa

University of Arizona

Tucson, AZ 85721

VAUGHAN F. R. Jones

University of California

Berkeley, CA 94720

STEVEN KerckHofF

Stanford University

Stanford, CA 94305

\section{C. MOORE}

University of California

Berkeley, CA 94720

Martin ScharlemanN

University of California

Santa Barbara, CA 93106

HAROLd STARK

University of California, San Diego

La Jolla, CA 92093
R. ARENS
E. F. BECKENBACH
F. WoLF
K. YoshidA
ASSOCIATE EDITORS

\begin{tabular}{ll}
\multicolumn{1}{c}{$(1906-1982)$} & \multicolumn{1}{c}{$(1904-1989)$} \\
SUPPORTING & INSTITUTIONS \\
UNIVERSITY OF ARIZONA & UNIVERSITY OF OREGON \\
UNIVERSITY OF BRITISH COLUMBIA & UNIVERSITY OF SOUTHERN CALIFORNIA \\
CALIFORNIA INSTITUTE OF TECHNOLOGY & STANFORD UNIVERSITY \\
UNIVERSITY OF CALIFORNIA & UNIVERSITY OF HAWAII \\
MONTANA STATE UNIVERSITY & UNIVERSITY OF TOKYO \\
UNIVERSITY OF NEVADA, RENO & UNIVERSITY OF UTAH \\
NEW MEXICO STATE UNIVERSITY & WASHINGTON STATE UNIVERSITY \\
OREGON STATE UNIVERSITY & UNIVERSITY OF WASHINGTON
\end{tabular}

The Supporting Institutions listed above contribute to the cost of publication of this Journal, but they are not owners or publishers and have no responsibility for its content or policies.

Mathematical papers intended for publication in the Pacific Journal of Mathematics should be in typed form or offset-reproduced (not dittoed), double spaced with large margins. Please do not use built up fractions in the text of the manuscript. However, you may use them in the displayed equations. Underline Greek letters in red, German in green, and script in blue. The first paragraph must be capable of being used separately as a synopsis of the entire paper. In particular it should contain no bibliographic references. Please propose a heading for the odd numbered pages of less than 35 characters. Manuscripts, in triplicate, may be sent to any one of the editors. Please classify according to the 1991 Mathematics Subject Classification scheme which can be found in the December index volumes of Mathematical Reviews. Supply name and address of author to whom proofs should be sent. All other communications should be addressed to the managing editor, or Elaine Barth, University of California, Los Angeles, California 90024-1555-05.

There are page-charges associated with articles appearing in the Pacific Journal of Mathematics. These charges are expected to be paid by the author's University, Government Agency or Company. If the author or authors do not have access to such Institutional support these charges are waived. Single authors will receive 50 free reprints; joint authors will receive a total of 100 free reprints. Additional copies may be obtained at cost in multiples of 50 .

The Pacific Journal of Mathematics (ISSN 0030-8730) is published monthly except for July and August. Regular subscription rate: $\$ 190.00$ a year (10 issues). Special rate: $\$ 95.00$ a year to individual members of supporting institutions.

Subscriptions, orders for numbers issued in the last three calendar years, and changes of address should be sent to Pacific Journal of Mathematics, P.O. Box 969, Carmel Valley, CA 93924, U.S.A. Old back numbers obtainable from Kraus Periodicals Co., Route 100, Millwood, NY 10546.

The Pacific Journal of Mathematics at P.O. Box 969, Carmel Valley, CA 93924 (ISSN 0030-8730) is published monthly except for July and August. Second-class postage paid at Carmel Valley, California 93924, and additional mailing offices. Postmaster: send address changes to Pacific Journal of Mathematics, P.O. Box 969, Carmel Valley, CA 93924.

PUBLISHED BY PACIFIC JOURNAL OF MATHEMATICS, A NON-PROFIT CORPORATION Copyright (C) 1991 by Pacific Journal of Mathematics 


\section{Pacific Journal of Mathematics}

Vol. 149, No. $2 \quad$ June, 1991

Manuel Alfaro Garcia, Mark Conger and Kenneth Hodges, The structure

of singularities in $\Phi$-minimizing networks in $\mathbf{R}^{2}$

Werner Balser, Dependence of differential equations upon parameters in

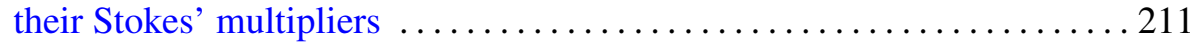

Enrico Casadio Tarabusi and Stefano Trapani, Envelopes of holomorphy

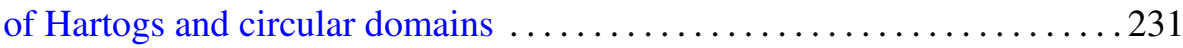

Hermann Flaschka and Luc Haine, Torus orbits in $G / P \ldots \ldots \ldots \ldots 251$

Gyo Taek Jin, The Cochran sequences of semi-boundary links .......... 293

Yasuyuki Kawahigashi, Cohomology of actions of discrete groups on

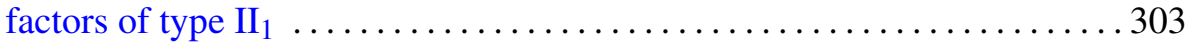

Ki Hyoung Ko and Lawrence Smolinsky, A combinatorial matrix in 3-manifold theory

W. B. Raymond Lickorish, Invariants for 3-manifolds from the combinatorics of the Jones polynomial

Peter Arnold Linnell, Zero divisors and group von Neumann algebras . . . . . 349

Bruce Harvey Wagner, Classification of essential commutants of abelian

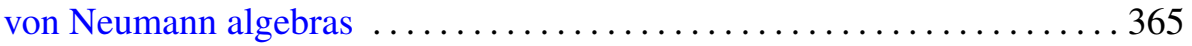

Herbert Walum, Multiplication formulae for periodic functions ..........383 